7. Christie C, Mazon D, Hierholzer W Jr, Patterson JE. Molecular heterogeneity of Acinetobacter baumannii isolates during seasonal increase in prevalence. Infect Control Hosp Epidemiol 1995;16:590-594.

8. Fukuta Y, Clarke LG, Shields RK, Wagener MM, Pasculle AW, Doi Y. Lack of seasonality in the occurrence of multidrug-resistant Acinectobacter baumannii complex. Infect Control Hosp Epidemiol 2012;33:1051-1052.
9. Cohen AL, Calfee D, Fridkin SK, et al. Recommendations for metrics for multidrug-resistant organisms in healthcare settings: SHEA/ HICPAC position paper. Infect Control Hosp Epidemiol 2008;29: 901-913.

10. Box GE, Jenkins GM, Reinsel CG. Time series analysis: forecasting and control. Hoboken NJ: Wiley; 2015.

\title{
Dissemination of Acinetobacter baumannii OXA-23 in old and new intensive care units without transfer of colonized patients
}

\author{
Rafael Renato Brondani Moreira MSc, Giselle Fukita Viana PhD, Amanda Carina Coelho de Moraes MD, \\ Marina de Souza Bastos PhD, Sheila Alexandra Belini Nishiyama PhD, Márcia Maria dos Anjos Szczerepa PhD, \\ Celso Luiz Cardoso PhD and Maria Cristina Bronharo Tognim PhD \\ Department of Basic Health Sciences, State University of Maringá, Maringá, Paraná, Brazil
}

To the Editor-The contamination of the environment and the hands of health professionals, transfer of patients, and movements of health professionals between hospitals are all possible routes for the dissemination of Acinetobacter baumannii. ${ }^{1-3}$ In our region during 2004-2008, an endemic carbapenem-resistant A. baumannii (CRAb) was detected. ${ }^{4}$ Later, it was verified (2011-2014), with a change in the dissemination mode of this microorganism (ie, the endemic situation to polyclonal dissemination). ${ }^{5}$ However, the routes of spread of $A$. baumannii have not yet been established.

In this study, we analyzed the effect of constructing a new ICU in a Brazilian hospital on the dissemination of A. baumannii. In the first 6 months, 22 clinical isolates were collected from an old ICU ( 12 beds), and in the next 6 months, 26 clinical isolates were collected from a newly installed ICU (24 beds).

In the new ICU, the presence of $A$. baumannii in the environment was investigated for a period of up to 15 days before and 15 days after patient admission to the unit. The samples were collected from bedside table, antiseptic dispenser, cardiac monitor, infusion pump, and bedrail, using sterile swabs moistened with sterile saline solution. Each swab was then used to inoculate a MacConkey agar plate.

The identification and antimicrobial susceptibility of bacterial isolates were assessed using a BD Phoenix system (Becton, Dickinson and Company, Franklin Lakes, NJ, USA). The minimum inhibitory concentrations of imipenem, meropenem, and polymyxin B were confirmed using the agar-dilution method. ${ }^{6}$

A multiplex PCR assay was performed to detect the presence of MBL genes ( $b l a_{\mathrm{IMP}}, b l a_{\mathrm{VIM}}, b l a_{\mathrm{GIM}}, b l a_{\mathrm{SPM}}$, and $\left.b l a_{\mathrm{SIM}}\right)$ and oxacillinase genes $\left(b l a_{\text {OXA23 }}, b l a_{\text {OXA24 }}, b l a_{\text {OXA51 }}\right.$, and $\left.b l a_{\text {OXA58 }}\right){ }^{7,8}$

Molecular typing was performed with enterobacterial repetitive intergenic consensus-polymerase chain reaction (ERIC-PCR) assays. Computer-assisted analysis was performed with BioNumerics version 6.5 software (Applied Maths, Sint-Martens-Latem, Belgium) with Dice correlation coefficient $\geq 0.93$. $^{9}$

Author for correspondence: Maria Cristina Bronharo Tognim, Laboratório de Microbiologia, Departamento de Ciências Básicas da Saúde, Universidade Estadual de Maringá, Avenida Colombo 5790, Maringá, Paraná, CEP 87020-900, Brasil. E-mail: mcbtognim@uem.br or cristinatognim@gmail.com

Cite this article: Moreira RRB et al. (2018) Dissemination of Acinetobacter baumannii OXA-23 in old and new intensive care units without transfer of colonized patients. Infection Control \& Hospital Epidemiology 2018, 39, 1135-1137. doi: 10.1017/ice.2018.168
In total, $48 \mathrm{~A}$. baumannii were isolated. The clinical and colonization isolates were obtained from tracheal aspirates $(n=26)$, urine $(n=6)$, blood $(n=5)$, cerebrospinal fluid $(n=1)$, wound secretion $(n=1)$, and nasal swabs $(n=4)$, oral swabs $(\mathrm{n}=4)$, and axillar swabs $(\mathrm{n}=1)$. During the study period, no patient infected or colonized with A. baumannii was transferred from the old to the new ICU, and no A. baumannii isolates were detected in the environment of the new ICU.

Of the 48 isolates, $65 \%$ and $50 \%$ were resistant to imipenem and meropenem, respectively. The most effective of the antibiotics tested was polymyxin B (100\% sensitivity), followed by tetracycline (73\%) and tobramycin (52\%). Comparing the isolates of A. baumannii from the 2 ICUs revealed an increase in resistance to imipenem in the isolates from the new unit (from 50\% to 69\%).

All isolates carried $b l a_{\mathrm{OXA} 51}$, and $29(60 \%)$ also carried $b l a_{\text {OXA23 }}$ (14 isolates from the old ICU and 15 isolates from the new unit). No strain was identified as a producer of MBL, OXA-58 or OXA-24.

The findings that $60 \%$ of the A. baumannii isolates showed the $b l a_{\text {OXA23 }}$ gene and that $11(38 \%)$ were susceptible to carbapenems can be explained. The expression of resistance depends not only on the presence of the $b l a_{\mathrm{OXA} 23}$ gene but also on its association with an insertion sequence, such as ISAbal, which enhances the expression of the $b a_{\text {OXA23 }}$ gene. These findings are worrisome because this insertion sequence may be inserted into a plasmid that has a high capacity for mobilization and dissemination. ${ }^{10}$

Molecular typing by ERIC-PCR of the $48 \mathrm{~A}$. baumannii isolates detected 17 different clusters (Fig. 1, A-Q). Two clusters were detected in both ICUs (I and J). Cluster J was detected in a patient admitted to the old ICU (negative for $b l_{a_{\mathrm{OXA} 23}}$ ) and in another patient admitted to the new ICU (positive for $b l a_{\text {OXA23 }}$ ). Cluster I was detected in 10 patients ( 5 in the old and 5 in the new ICU). This cluster was identical to the endemic CRAb detected in our region. ${ }^{5}$

The old ICU showed no distinct clusters, and the new ICU had 10 clusters. The most common clusters were $\mathrm{B}, \mathrm{I}$, and $\mathrm{H}$, which comprised $\sim 50 \%$ (23 of 48 ) of all A. baumannii isolates. Clusters B and $\mathrm{H}$ were isolated only in the new ICU, while cluster I was detected in both units. All clusters showed at least 1 isolate carrying $b l a_{\text {OXA23 }}$.

Molecular typing revealed a certain degree of clonal diversity, and despite the construction of the new ICU, several $A$. baumannii-producing OXA-23 coexist, making control more 


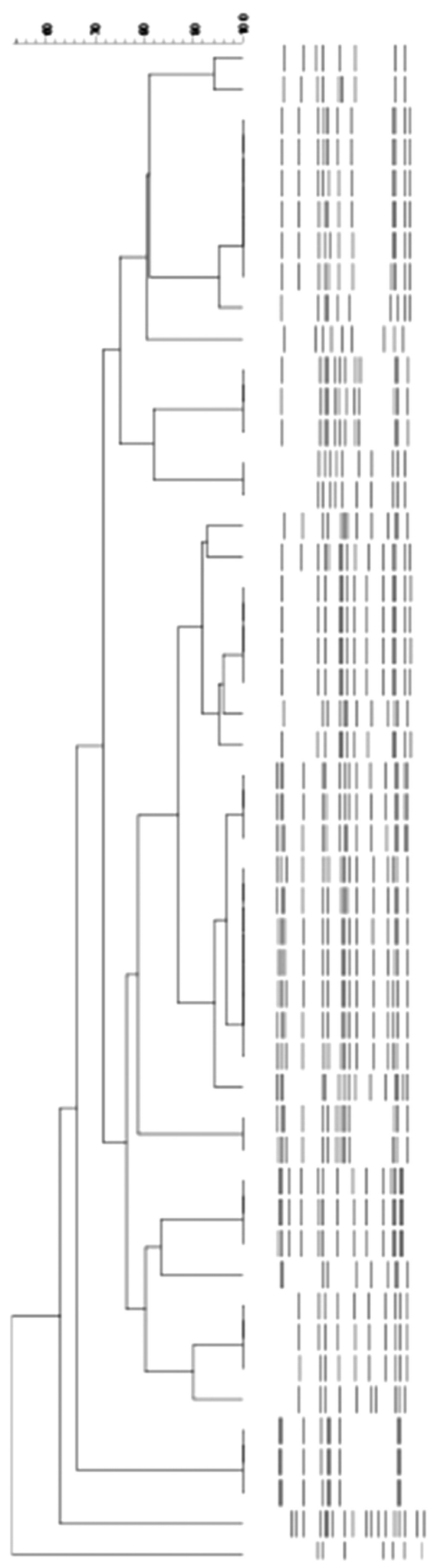

\begin{tabular}{|c|c|}
\hline lsolates & Sample type \\
\hline $\operatorname{SCAC} 27$ & Adtary swabs \\
\hline $\operatorname{SCAC} 28$ & Ume \\
\hline $\operatorname{SCAC} 16$ & Tracheal secretion \\
\hline SCAC 15 & Tracheal secretion \\
\hline $\operatorname{SCAC} 19$ & Tracheal secretion \\
\hline $\operatorname{SCAC} 20$ & Tracheal secretion \\
\hline $\operatorname{SCAC} 21$ & surgical secretion \\
\hline $\operatorname{SCAC} 22$ & Ume \\
\hline $\operatorname{SCAC} 17$ & Ume \\
\hline $\operatorname{SCAC} 26$ & Oral swabs \\
\hline $\operatorname{SCAb} 30$ & Tracheal secretion \\
\hline $\operatorname{SCAb} 29$ & Tracheal secretion \\
\hline $\operatorname{SCAD} 31$ & Nasal swabs \\
\hline $\operatorname{ScAb} 28$ & Hemocuture \\
\hline$S C A b 16$ & LCR \\
\hline $\operatorname{sc}$ ac 7 & Oral swabs \\
\hline $\operatorname{SCAC} 18$ & Tracheal secretion \\
\hline $\operatorname{SCAC} 11$ & Hemocuture \\
\hline $\operatorname{SCAC} 12$ & Hemocuture \\
\hline $\operatorname{SCAC} 13$ & Tracheal secretion \\
\hline $\operatorname{SCAC} 14$ & Tracheal secretion \\
\hline $\operatorname{SCAC} 6$ & Nasal owabs \\
\hline $\operatorname{SCAC} 10$ & Tracheal secretion \\
\hline $\operatorname{SCAC} 2$ & Tracheal secretion \\
\hline $\operatorname{SCAC} 3$ & Tracheal secretion \\
\hline $\operatorname{SCAC~} 4$ & Tracheal secretion \\
\hline \multicolumn{2}{|c|}{ Endemic dor } \\
\hline $\operatorname{SCAC} 8$ & Ume \\
\hline $\operatorname{SCAD} 33$ & Tracheal secretion \\
\hline$S C A b 34$ & Hembauture \\
\hline$S C A b 35$ & Tracheal secretion \\
\hline$S C A b 03$ & Hemocuture \\
\hline $\operatorname{ScAb} 27$ & Une \\
\hline $\operatorname{SCAC} 1$ & Tracheal secretion \\
\hline $\operatorname{SCAC} 9$ & Tracheal secretion \\
\hline$S C A b 32$ & Tracheal secretion \\
\hline $\operatorname{SCAb} 1$ & Tracheal secretion \\
\hline$S C A B C$ & Tracheal secretion \\
\hline $\operatorname{ScAb} 5$ & Tracheal secretion \\
\hline $\operatorname{SCAC} 5$ & Oral swabs \\
\hline $\operatorname{SCAb} 19$ & Tracheal secretion \\
\hline $\operatorname{SCAb} 21$ & Uthe \\
\hline SCAD 23 & Tracheal secretion \\
\hline$S C A b 26$ & Tracheal secretion \\
\hline$S C A b 8$ & Nasal swabs \\
\hline$S C A b 7$ & Une \\
\hline $\operatorname{SCAb} 9$ & Nasal swabs \\
\hline$S C A D 25$ & Tracheal secretion \\
\hline $\operatorname{ScAC} 25$ & Oral swabs \\
\hline
\end{tabular}

$M i C(\mu / m L)$

\begin{tabular}{|c|c|c|c|c|}
\hline IMP & MEM & PMB & OXA-gene & QUSTER \\
\hline 16 & 16 & 0.5 & $51-23$ & A \\
\hline 8 & 8 & 0.5 & 51 & $A$ \\
\hline 8 & 16 & 0.5 & $51-23$ & $B$ \\
\hline 8 & 16 & 1 & 51 & B \\
\hline 16 & 16 & 1 & 51 & B \\
\hline 16 & 16 & 1 & $51-23$ & B \\
\hline 8 & 16 & 1 & $51 \cdot 23$ & $B$ \\
\hline 16 & 8 & 1 & $51-23$ & 8 \\
\hline 8 & 16 & 1 & $51 \cdot 23$ & $B$ \\
\hline 1 & 0.5 & 0.5 & 51 & $c$ \\
\hline 8 & 8 & 0.5 & 51.23 & 0 \\
\hline 8 & 8 & 1 & 51,23 & 0 \\
\hline 8 & 8 & 1 & 51 & 0 \\
\hline 8 & 16 & 0.5 & 51 & $\mathbf{E}$ \\
\hline 8 & 16 & 0.5 & 51 & $\mathbf{E}$ \\
\hline 8 & 1 & 1 & 51 & $F$ \\
\hline 2 & 2 & 1 & $51-23$ & $F$ \\
\hline 16 & 16 & 1 & 51 & 0 \\
\hline 4 & 1 & 1 & $51-23$ & G \\
\hline 8 & 2 & 1 & 51 & G \\
\hline 8 & 1 & 1 & 51 & G \\
\hline 8 & 1 & 1 & 51,23 & $H$ \\
\hline 8 & 8 & 1 & $51-23$ & 1 \\
\hline 1 & 1 & 1 & $51-23$ & $J$ \\
\hline 4 & 4 & 1 & 51,23 & $J$ \\
\hline \multirow[t]{2}{*}{2} & 1 & 1 & - & $J$ \\
\hline & & & & $J$ \\
\hline 2 & 1 & 1 & 51,23 & $J$ \\
\hline 0.5 & 2 & 1 & 51.23 & $J$ \\
\hline 1 & 2 & 1 & 51,23 & $J$ \\
\hline 1 & 2 & 1 & 51.23 & $J$ \\
\hline 2 & 1 & 1 & 51 & $J$ \\
\hline 0.5 & 1 & 1 & 51 & $J$ \\
\hline 2 & 4 & 1 & 51 & $J$ \\
\hline 8 & 8 & 1 & 51,23 & $\kappa$ \\
\hline 1 & 8 & 1 & 51 & K \\
\hline 2 & 2 & 1 & 51 & L \\
\hline 1 & 2 & 1 & $51-23$ & $L$ \\
\hline 8 & 8 & 1 & 51 & $L$ \\
\hline 8 & 1 & 1 & 51,23 & $M$ \\
\hline 1 & 2 & 1 & 51 & $N$ \\
\hline 1 & 1 & 1 & 51,23 & $N$ \\
\hline 8 & 2 & 2 & 51,23 & $N$ \\
\hline 1 & 2 & 1 & 51,23 & $N$ \\
\hline 8 & 8 & 0.5 & $51-23$ & 0 \\
\hline 8 & 8 & 1 & $51 \cdot 23$ & 0 \\
\hline 8 & 8 & 1 & $51-23$ & 0 \\
\hline 8 & 0.125 & 1 & 51.23 & $P$ \\
\hline 8 & 0.5 & 2 & 51 & 0 \\
\hline
\end{tabular}

Fig. 1. Dendrogram, minimum inhibitory concentration (MIC) for imipenem (IMP), meropenem (MEM), and polymyxin B (PMB) of $A$. baumannii isolates from 2ICUs of Brazilian hospitals. Isolates from the old ICU are designated "SCAb," and isolates from the new ICU are designated "SCAc."

difficult. Moreover, the same endemic cluster persisted in both ICUs, that is, it was transferred to a completely new ICU.

Although many studies have demonstrated the importance of environmental contamination in the dissemination of pathogens, ${ }^{1-3}$ our study showed that an environment free of contamination was not sufficient to control either the dissemination of an endemic carbapenem-resistant or the emergence of new clusters of $A$. baumannii. 
The presence of cluster I in both ICUs, identical to the endemic cluster described by Saalffeld et $\mathrm{al}^{4}{ }^{4}$ demonstrates the importance of interhospital dissemination. Although the environment was not the main cause of dissemination, we believe that the contamination of the hands of healthcare professionals may have contributed to the dissemination of $A$. baumannii isolates, and the failure to verify this dissemination route was a limiting factor in our study.

Our study showed that after the ICU was re-established in a new building (ie, a new ICU), the dissemination of endemic clone-producing OXA-23 was maintained even though the new ICU environment was not contaminated. This occurrence demonstrates that additional measures are required to control the dissemination of this important hospital pathogen.

Acknowledgments. We thank Dr Janet W. Reid (JWR Associates) for editing the English text.

Financial support. No financial support was provided relevant to this article.

Conflicts of interest. All authors report no conflicts of interest relevant to this article.

\section{References}

1. Harding CM, Hennon SW, Feldman MF. Uncovering the mechanisms of Acinetobacter baumannii virulence. Nat Revs 2018;16:91-102.

2. Greene C, Vadlamudi G, Newton D, Foxman B, Xi C. The influence of biofilm formation and multidrug resistance on environmental survival of clinical and environmental isolates of Acinetobacter baumannii. Am J Infect Control 2016;44:e65-e71.

3. Weber DJ, Rutala WA. Understanding and preventing transmission of healthcare-associated pathogens due to the contaminated hospital environment. Infect Control Hosp Epidemiol 2013;34:449-452.

4. dos Santos Saalfeld SM, Viana GF, Dias Siqueira VL, Cardoso CL, Garcia LB, Tognim MCB. Endemic carbapenem-resistant Acinetobacter baumannii in a Brazilian intensive care unit. J Hosp Infect 2009;72: 365-368.

5. Viana GF, Zago MCB, Menegucci TC, Zarpellon MN, Nishiyama SAB, Cardoso CL, Tognim MCB. Change in the dissemination mode of multidrug-resistant Acinetobacter baumannii isolates after implementation of control measures. J Hosp Infect 2016;93:217-220.

6. Clinical and Laboratory Standards Institute. 2017 Performance standards for antimicrobial susceptibility testing. CLSI document M100. 27th ed. Wayne, PA: CLSI 2017.

7. Ellington MJ, Kistler J, Livermore DM, Woodford N. Multiplex PCR for rapid detection of genes encoding acquired metallo- $\beta$-lactamases. J Antimicrob Chemother 2007;59:321-322.

8. Woodford N, Ellington MJ, Coelho JM, et al. Multiplex PCR for genes encoding prevalent OXA carbapenemases in Acinetobacter spp. Int $J$ Antimicrob Agent 2006;27:351-353.

9. Silbert S, Pfaller MA, Hollis RJ, Barth AL, Sader HS. Evaluation of three molecular typing techniques for nonfermentative gram-negative bacilli. Infect Control Hosp Epidemiol 2004;25:847-851.

10. Turton JF, Ward ME, Woodford N. The role of ISAbal in expression of OXA carbapenemase genes in Acinetobacter baumannii. FEMS Microbiol Lett 2006;258:72-77.

\title{
Extended-Spectrum Beta-Lactamase (ESBL)-Producing Escherichia coli versus Klebsiella pneumoniae: Does type of germ really matter?
}

\author{
Benjamin Davido MD, $\mathrm{MSc}^{1}$, Pierre de Truchis $\mathrm{MD}^{1}$, Christine Lawrence PharmD ${ }^{2}$ and Aurélien Dinh MD, MSc ${ }^{1}$ \\ ${ }^{1}$ Maladies Infectieuses, Hôpital Universitaire Raymond-Poincaré, AP-HP, Garches, France; and ${ }^{2}$ Microbiologie, Hôpital Universitaire Raymond-Poincaré, AP-HP, \\ Garches, France
}

To the Editor-We read with great interest the recent article by Scheuerman et $\mathrm{al}^{1}$ showing that extended-spectrum $\beta$-lactamase (ESBL)-producing Escherichia coli (ESBL-EC) and Klebsiella pneumoniae (ESBL-KP) bloodstream infections (BSIs) differ significantly in terms of mortality $(33.7 \%$ vs $17.4 \% ; P=.016)$. Because their study concerns a highly relevant and popular topic, some points should be discussed.

First, ESBL-KP-infected patients were more often hospitalized in ICU than those infected by ESBL-EC $(P<.001)$, partly due to a septic shock, which may explain such a high rate of mortality (33.7\%) for a bloodstream infection (BSI). Indeed, the observed mortality rate for ESBL-KP was similar to the average mortality for those with gram-negative BSIs in the ICU (35\%) according to

\footnotetext{
Author for correspondence: B. Davido, MD, MSc, Maladies Infectieuses, Hôpital Universitaire Raymond-Poincaré, Garches 92380, France E-mail: benjamin.davido@aphp.fr

Cite this article: Davido B, et al. (2017). Extended-Spectrum Beta-Lactamase (ESBL)Producing Escherichia coli versus Klebsiella pneumoniae: Does type of germ really matter? Infection Control \& Hospital Epidemiology 2018, 39, 1137-1138. doi: 10.1017/ice.2018.152

the prospective EUROBACT International cohort study. ${ }^{2}$ Also, ICU-acquired BSIs are associated with a $40 \%$ increase in the risk of 30-day mortality. ${ }^{3}$ Therefore, it is hard to believe that such a difference could be accounted for in any statistical adjustment, and thus, it constitutes a selection bias.

Second, the main source of BSI was urinary tract in the ESBLEC arm $(P=.005)$, while it is acknowledged that the severity of urinary tract infection is not related to the presence of bacteremia. ${ }^{4}$ Such data underly the hypothesis that ESBL-KP infections might have been more severe than those due to ESBL-EC. For instance, multidrug-resistant BSIs complicating respiratory tract infections have been associated with an increased mortality (odds ratio [OR], 3.26; 95\% confidence interval [CI], 1.29-8.22). ${ }^{7}$

Third, no information is provided about the respective antimicrobial regimens between ESBL-EC and ESBL-KP patients. However, it is currently argued that carbapenem alternatives are associated with a higher mortality rate than carbapanems for the treatment of ESBL BSI. In fact, the MERINO trial by Harris et $\mathrm{al}^{5}$ was recently suspended due to an increase in mortality in the arm 